PROCEEDINGS OF THE AMERICAN MATHEMATICAL SOCIETY

Volume 126, Number 3, March 1998, Pages 913-915

S 0002-9939(98)04083-0

\title{
A GENERALIZATION OF BANCHOFF'S TRIPLE POINT THEOREM
}

\author{
P. AKHMETIEV, R. RIMÁNYI, AND A. SZÜCS \\ (Communicated by Thomas Goodwillie)
}

\begin{abstract}
Consider an immersion of a surface into $S^{3}$. Banchoff's theorem states that the parity of the number of triple points and the parity of the Euler characteristic of the surface coincide. Here we generalize this theorem to codimension 1 immersions of arbitrary even dimensional manifolds in spheres. The proof is an analogue of a proof of Banchoff's theorem circulated in preprint form due to R. Fenn and P. Taylor in 1977.
\end{abstract}

Let us consider a codimension 1 smooth generic (i.e. self-transverse) immersion $f$ of a closed manifold $M^{n}$ in the sphere $S^{n+1}$. Let us recall how a neighborhood of an $i$-tuple point (in $R^{n+1} \subset S^{n+1}$ ) looks like in such a self-transverse immersion. Consider the coordinate hyperplanes in $R^{i}$ and take the direct product of this configuration with $R^{n+1-i}$. What is obtained is diffeomorphic to the neighborhood of an $i$-tuple point in the image of $f$.

For any natural number $i, 1 \leq i \leq n+1$, let us denote by $\tilde{\Delta}_{i}$ the set of $i$-tuple points in $S^{n+1}$, i.e.

$$
\tilde{\Delta}_{i}=\left\{y \in S^{n+1} \mid f^{-1}(y) \text { consists of } i \text { different points }\right\} \text {. }
$$

As is well known, $\operatorname{dim} \tilde{\Delta}_{i}=n+1-i$, and $\bigcup_{r=i}^{\infty} \tilde{\Delta}_{r}$ is an immersed manifold (although it is not in general position, i.e. it is the image of a non-self-transverse immersion). Let $\Delta_{i}$ be a closed manifold such that $\bigcup_{r=i}^{\infty} \tilde{\Delta}_{r}$ is the image of an immersion of $\Delta_{i}$ in $S^{n+1}$.

Remark. Of course, many different manifolds can be immersed into $S^{n+1}$ so that their images are $\bigcup_{r=i}^{\infty} \tilde{\Delta}_{r}$. For example if a possible $\Delta_{i}$ is given, then any of its finite coverings serves as well. We make the choice of $\Delta_{i}$ explicit by assuming that the $i$-tuple points of $f$ are non-multiple points of the immersion $\Delta_{i} \uparrow S^{n+1}$.

We shall call the manifold $\Delta_{i}$ the $i$-tuple manifold of $f$. Our theorem claims that for $n$ even the sum of the Euler characteristics of $i$-tuple manifolds is even. (For $n=2$ this is exactly Banchoff's theorem.)

Received by the editors July 4, 1995 and, in revised form, September 2, 1996.

1991 Mathematics Subject Classification. Primary 57R42.

Key words and phrases. Immersion, multiple points.

The first author was supported in part by the Russian Foundation for Fundamental Research grant N 96-01-01166a. The second and the third authors were supported by the Hungarian National Science Foundation, Grant No. F-014906 and 4232, respectively.

(C)1998 American Mathematical Society 
Theorem. If $n>0$ is even, then

$$
\sum_{i=1}^{n+1} \chi\left(\Delta_{i}\right) \equiv 0 \bmod 2 .
$$

The following proof is an analogue of the proof in [FT] for Banchoff's triple point theorem.

Proof. Since $n$ is even, we can omit the terms corresponding to even $i$ 's, because in those cases the dimension of $\Delta_{i}$ is odd. Now let us triangulate the image of $f$ in such a way that for any $i$ the set of points of multiplicity $i$ or higher forms a subcomplex of $f(M)$.

Let $\alpha_{r}^{i}$ denote the number of $i$-dimensional simplexes whose interiors lie in $\tilde{\Delta}_{r}$, and let

$$
\beta_{r}=\alpha_{r}^{0}-\alpha_{r}^{1}+\ldots \pm \alpha_{r}^{n+1-r} .
$$

Observe that $\beta_{r}$ is not the Euler characteristic of any complex. However, we have that

$$
\chi\left(\Delta_{i}\right)=\sum_{r=i}^{n+1}\left(\begin{array}{l}
r \\
i
\end{array}\right) \beta_{r} .
$$

The coefficient $\left(\begin{array}{c}r \\ i\end{array}\right)$ counts the multiplicity of the self-intersection of $\Delta_{i}$ at $\tilde{\Delta}_{r}$. So

$$
\sum_{i=1}^{n+1} * \chi\left(\Delta_{i}\right)=\sum_{i=1}^{n+1} * \sum_{r=i}^{n+1}\left(\begin{array}{l}
r \\
i
\end{array}\right) \beta_{r}
$$

where ${ }^{*}$ indicates that the sum is taken only for odd $i$ 's. After changing the order of the summations we get:

$$
\sum_{r=1}^{n+1}\left(\sum_{i=1}^{r} *\left(\begin{array}{l}
r \\
i
\end{array}\right)\right) \beta_{r}=\sum_{r=1}^{n+1} 2^{r-1} \beta_{r} \equiv \beta_{1} \bmod 2 .
$$

Now let us paint the complement of $f(M)$ in $S^{n+1}$ in two colors in a chessboardstyle, i.e. let any two neighboring domains have different colors (where "neighboring" means that they are separated by a component of $\tilde{\Delta}_{1}$ ). This is possible, since $H_{n}\left(S^{n+1} ; Z_{2}\right)=0$.

Let $N$ be the boundary of an $\varepsilon$-neighborhood of $f(M)$ in the black subset of $S^{n+1}$. Notice that from the given triangulation of $f(M)$ we can construct a triangulation of $N$ by pushing the simplexes from $f(M)$ to $N$ in a reasonable way. Simplexes in $\tilde{\Delta}_{i}$ will have $2^{i-1}$ counterparts in $N$ ( $i$ hyperplanes divide the Euclidean $n$-space into $2^{i}$ parts, half of which are black). Thus:

$$
\chi(N)=\sum_{i=1}^{n+1} 2^{i-1} \beta_{i} \equiv \beta_{1} \bmod 2 .
$$

But $\chi(N)$ is even, because $N$ is embedded in codimension 1 (and $n>0$ ), so the proof is complete.

Remark 1. As is clear from the proof, the space $S^{n+1}$ can be replaced by any manifold such that its $n$th $Z_{2}$-homology group is 0 . 
Remark 2. The above proof does not work for $n$ odd, since the sum $\sum_{i=1}^{r} *\left(\begin{array}{l}r \\ i\end{array}\right)$ (where the star this time means summation for even $i$ 's) equals to $2^{r-1}-1$, so the sum in formula (1) gives $\sum_{r=2}^{n+1} \beta_{r}$ (which is clearly the Euler characteristic of the complex $f(M))$.

The figure 8 immersion of the circle in the plane shows that the statement of the theorem is false for $n=1$. A theorem of Freedman $[\mathrm{F}]$ (and its generalization to unoriented 3-manifolds given in [A]) shows that it is true for $n=3$. We do not know whether it is true or not for $n>3$.

Remark 3 . If we consider only oriented $n$-manifolds and their codimension 1 immersions in $S^{n+1}$, and the $n$th stable homotopy group of spheres has no 2-primary torsion, then the Euler characteristics of the $i$-tuple manifolds are all even, for any $i$. (Indeed, for any $i, \chi\left(\Delta_{i}\right)$ mod 2 defines a homomorphism from the stable homotopy group $\pi_{n+N}\left(S^{N}\right), N>>n$ to $Z_{2}$.)

In particular the statement of the theorem is true for $n=5$ or $n=13$ for oriented manifolds.

Remark 4. If the dimension $n=4$, then more is true than is stated in the theorem, namely all $\chi\left(\Delta_{i}\right)$ 's are even, since the stable homotopy group $\pi_{5}^{s}\left(R P^{\infty}\right)$ vanishes (see $[\mathrm{L}])$, and this group is isomorphic to the cobordism group of immersions of 4-manifolds into $R^{5}$.

\section{REFERENCES}

[A] P. Akhmetiev, An elementary proof of Freedman's theorem on immersions, St. Petersburg Math. J. 7 (1996), 749-754.

[B] T. Banchoff, Triple points and surgery of immersed surfaces, Proc. Amer. Math. Soc. 46 (1974), 407-413. MR 51:14066

[F] M. Freedman, Quadruple points of 3-manifolds in $S^{4}$, Comment. Math. Helvetici 53 (1978), 385-394. MR 81b:57027

[FT] R. Fenn, P. Taylor, On the number of triple points of an immersed surface, (unpublished) preprint (1977).

[L] A. Liulevicius, A theorem in homological algebra and stable homotopy of projective spaces, Trans. Amer. Math. Soc., 109 (1963), 540-552. MR 27:6270

Institute of Terrestrial Magnetism and Radio Wave Propagation, Russian Academy of Sciences, Troitsk, Moscow Region 142092, Russia

ELTE Department of Geometry, Budapest, Rákóczi út 5., 1088, Hungary

Elte Department of Analysis, Budapest, Múzeum krt. 6-8, 1088, Hungary

E-mail address: szucsandras@eudens.elte.hu 